\title{
FABRICATION OF HIGHLY POROUS SCAFFOLD MATERIALS BASED ON FUNCTIONALIZED OLIGOLACTIDES AND PRELIMINARY RESULTS ON THEIR USE IN BONE TISSUE ENGINEERING
}

\author{
S. Vogt ${ }^{1}$, Y. Larcher ${ }^{2}$, B. Beer ${ }^{1}$, I. Wilke ${ }^{2}$ and M. Schnabelrauch ${ }^{1 *}$ \\ ${ }^{1}$ INNOVENT Technologieentwicklung, Jena, Germany \\ ${ }^{2}$ Research Centre of Medical Technology and Biotechnology, Bad Langensalza, Germany
}

\begin{abstract}
Tissue engineering offers a promising new approach to repair bone defects. Its practical realisation is connected with the development of suitable scaffold materials. In the present work, functionalized oligolactides have been prepared and used as macromers for the scaffold fabrication. The developed fabrication process leads to highly porous scaffolds, available in various shapes and sizes, with an open interconnective pore structure and porosities up to $90 \%$. Degradable or even osteoconductive components as well as biocompatible comonomers can be used as additives to modulate the scaffold properties. Under in vitro conditions, the scaffolds exhibit a continuous degradation with varying degradation rates depending on their material composition. In vitro studies on the cultivation of osteoblasts on the scaffolds were performed and revealed their excellent biocompatibility. Cell growth on the scaffold surfaces and inside the scaffolds, formation of extracellular matrix and starting mineralization were detected by microscopical and histological analyses. Based on these results the developed materials are well-suited candidates for the design of tailor-made matrices in bone tissue engineering.
\end{abstract}

Key Words: Scaffold, resorbable polymer, polylactide, osteoblast, tissue engineering

*Address for correspondence:

Matthias Schnabelrauch

INNOVENT Technologieentwicklung e. V.

Pruessingstr. 27B

D-07745 Jena, Germany

FAX number : +49-3641-282530

E-mail: ms@innovent-jena.de

\section{Introduction}

Bone tissue engineering represents one of the most promising concepts in orthopaedic surgery for bone repair and reconstruction (Boyan et al., 1999; Griffith and Naughton, 2002; Jackson and Simon, 1999). It may potentially provide suitable filler materials for large orthopaedic defects and implants with better mechanical properties and ingrowth behaviour than those used currently. A key role in this concept plays the biodegradable scaffold. This threedimensional construct serves as a support for the osteoblasts to proliferate and maintain their differentiated function and it determines the shape and size of the new bone.

A variety of materials based on synthetic and naturally occurring polymers, ceramics and bioglasses have been investigated for bone tissue engineering (Burg et al., 2000; Fleming et al., 2000; Simske et al., 1997). Nevertheless, there is still an urgent need for scaffolds with characteristics that are properly fitted to the special demands in tissue engineering (Luyten et al., 2001; Hench and Polak, 2002). Among the degradable polymers, polylactones, especially polylactides, polyglycolides and their copolymers, have found considerable interest as scaffold materials (Agrawal et al., 1997; Hutmacher, 2000; Middleton and Tipton, 2000). This class of polymers offers the possibility to tailor material properties that are quite important both for the scaffold fabrication technologies and the scaffold functions. In addition to a simple variation of the polymerization conditions, tailoring can be carried out by varying several factors like the monomer composition, the type of used initiator, the employed lactone : initiator ratio, and by a subsequent end group functionalization of the synthesized polylactone. The latter method permits for example, chain extension (Lendlein et al., 1998) or cross-linking of polylactones (Lee and Kim, 1997; Storey et al., 1993; Wenz and Nies, 1996) by introducing reactive functional groups at the ends of the polymer chains. Methacrylate-endcapped oligo-D,L-lactides have been prepared in this way by the trimethylolpropaneinitiated ring-opening oligomerization of D,L-lactide and reaction of the formed triol with methacryloyl chloride (Storey et al., 1993). The obtained prepolymers can be used as resin components for the fabrication of biodegradable composites. Recently, acrylated oligolactides emanating from glycerol have been synthesized and photocrosslinked with poly(ethylene gycol)-monoacrylate to form polymer networks usable as scaffolds for cell cultivation (Han and Hubbell, 1997). Films obtained from this material were biodegradable and displayed a high resistance to undesired cell adhesion. 
From our point of view, crosslinked polylactones have several advantages compared to non-crosslinked ones. Devices of defined shape and size, made from common polylactones can be fabricated by thermal or solvent-based processes. Thermal processing like injection moulding needs relatively expansive equipment as well as careful adjustment of the processing temperature and exclusion of moisture to avoid polymer degradation. Most of the suitable solvents for polylactones, namely dichloromethane, chloroform or tetrahydrofurane are highly toxic for cells. Therefore, after processing polylactones from their solutions, the solvents have to be completely removed, which is often a time-consuming procedure for polymers. Using crosslinkable oligolactone macromers, which are liquids at room or body temperature, it is possible to remove the necessary solvents more easily before the polymerization occurs. In contrast to conventional polylactides, solventfree compositions of liquid oligolactide macromers can be used to design injectable compositions, which fill out an existing bone defect and form a biodegradable implant by in situ polymerization similar to known non-biodegradable bone cements. A further advantage of crosslinked polylactones compared to non-crosslinked ones is their higher mechanical stability. Especially, if highly porous scaffolds with inter-connective macropores are fabricated from common linear polylactones, the compressive strength of these devices is often not satisfactory for use in bone engineering and also further shaping of the porous materials by usual mechanical procedures tends to becomes a critical point.

Therefore our interest was focused on thermally crosslinkable, methacrylate containing oligolactide macromers serving as matrix components for the generation of polymer networks and composites. It was our hypothesis that such polymeric materials, consisting of methacrylate and lactate building blocks, have a comparably good biocompatibility like polylactides and polymethacrylates alone.

In this paper, we wish to report on the synthesis and characterisation of new oligo-L-lactide macromers based on a dianhydro-D-glucitol-oligo-L-lactide endcapped with methacrylate groups. Using this macromer we developed a simple fabrication procedure based on a curing and a subsequent leaching step. Polymer network and composite scaffolds have been prepared from compositions of the oligoL-lactide macromer containing various degradable additives. The properties of the resulting scaffolds are studied and first results on the cultivation of osteoblasts on the fabricated scaffolds are presented.

\section{Materials and Methods}

\section{Educts and analytical methods}

L- and D,L-lactide (Boehringer Ingelheim, Germany) were dried in vacuum prior to use. All other reagents and solvents used were dried and purified by standard methods (Perrin and Amarego, 1988). Cell culture materials were obtained from Sigma Chemie (Taufkirchen, Germany) except fetal calf serum (fcs) which was obtained by HyClone (Logan, UT, USA). The KBr technique was used for per- forming infra-red (IR) spectroscopy. Gel permeation chromatography (GPC) was carried out in chloroform on a Jasco (Gross-Umstadt, Germany) device. Molecular weights were calculated related to polystyrene standards. A Topcon (Tokyo, Japan) SM 510 scanning electron microscope was used for studying the pore structure of the porous scaffolds.

\section{Oligolactide synthesis}

The synthesis of the oligo-L-lactide DL-1 (Fig. 1, n=2) is given as a representative procedure. A mixture of dianhydro-D-glucitol (7.3 g, $0.05 \mathrm{~mol}$ ), L-lactide (14.41 $\mathrm{g}, 0.10 \mathrm{~mol})$, and stannous ethylhexanoate $(0.09 \mathrm{~g}, 0.22$ mmol) was stirred under nitrogen and exclusion of moisture at $140^{\circ} \mathrm{C}$ for $4 \mathrm{~h}$. The melt was allowed to cool and was dissolved in dichloromethane $(40 \mathrm{ml})$. The solution was filtrated and the oligolactide was precipitated by pouring into heptane $(400 \mathrm{ml})$. Further purification was achieved by repeating the latter process several times. Finally, the isolated oligolactide (20.4 g, yield $94 \%$ ) was dried in vacuum at $25^{\circ} \mathrm{C}$ to constant weight.

The oligo-L-lactides DL-2 (yield $92 \%$ ) and DL-3 (yield $96 \%$ ) were prepared following the described procedure except that $4 \mathrm{~mol}$ and $6 \mathrm{~mol}$, respectively, of L-lactide per mol dianhydro-D-glucitol were used.

\section{Macromer preparation}

The macromer MDL-1 was synthesized as follows: DL-1 (20.4 g) was dissolved in dichloromethane $(40 \mathrm{ml})$. After the addition of triethylamine $(52.3 \mathrm{ml}, 0.38 \mathrm{~mol})$, methacryloyl chloride $(27.3 \mathrm{ml}, 0.28 \mathrm{~mol})$ was slowly added with stirring at $0-5^{\circ} \mathrm{C}$ under moisture exclusion. The mixture was brought to room temperature and stirring was continued overnight. A further quantity of dichloromethane $(20 \mathrm{ml})$ was added and the reaction mixture was successively extracted several times with $1 \mathrm{M} \mathrm{HCl}(50 \mathrm{ml})$, saturated aqueous solution of $\mathrm{NaHCO}_{3}(100 \mathrm{ml})$, and with distilled water. The organic phase was dried over $\mathrm{Na}_{2} \mathrm{SO}_{4}$, filtrated and treated with silica gel (Merck 60) to remove coloured impurities. A few crystals of $p$-methoxyphenol were added to the filtrated macromer solution and the solvent was removed under reduced pressure. Drying of the resulting residue in vacuum produced MDL-1 as a slightly yellow oil (yield: $16.7 \mathrm{~g}, 62 \%$ ).

The same procedure was used for the acylation of DL2 and DL-3, resp., producing MDL-2 (yield $56 \%$ ) and MDL-3 (yield $45 \%$ ) as yellowish viscous oils.

\section{Scaffold fabrication}

The porogen matrix was produced from saccharose. A suspension of saccharose crystals in a saturated aqueous saccharose solution was poured into silicon moulds possessing a cylindrical hollow, $30-50 \mathrm{~mm}$ in diameter and $5-10 \mathrm{~mm}$ in depth. After pressing the material with an appropriate plug, the filled moulds were dried in an exsiccator to obtain slices of the desired porogen matrices.

The produced slices were soaked with the corresponding macromer mixture (composition of the macromer mixture is given in Table 2). The impregnated slices were cured at $100^{\circ} \mathrm{C}$ for $1 \mathrm{~h}$ in a drying oven. Small discs, $10 \mathrm{~mm}$ in diameter and $5 \mathrm{~mm}$ in height were fabricated from the cured slices using a drilling machine with a hollow drill. Subse- 
quently, the discs were stored in distilled water for $16 \mathrm{~h}$ to leach out the sucrose. During this time the water is changed several times. The resulting porous discs were treated twice with boiling distilled water for $1 \mathrm{~h}$ and dried at $70^{\circ} \mathrm{C}$ for 18 h.

\section{Porosity estimation}

The porosities of the highly porous scaffolds were calculated from the apparent densities of the scaffolds and the densities of the solid polymeric material (Thomson et al., 1995). The weight, height, and diameter of the scaffolds were determined to calculate the apparent densities. The determination of the densities of the solid polymeric materials was performed with a pycnometer. For this purpose finely ground material of the scaffold was used. For each sample five specimens were tested.

\section{Degradation studies}

Degradation studies were carried out at $37^{\circ} \mathrm{C}$ in a simulated body fluid (SBF) medium (Cho et al., 1995), which was replaced every week. Cylindrical samples of the scaffolds, $10 \mathrm{~mm}$ in diameter and $5 \mathrm{~mm}$ in height, were run in duplicate. Each week samples were taken to determine the weight loss and the compression strength of the scaffolds.

\section{Compression strength}

A tensile testing machine (Instron, 4467) was used for the measurement of the compression strength. Cylindrical samples, having the same dimensions as described above for the degradation studies were prepared for the mechanical testing. In a first series of experiments the compression strength of the samples was measured immediately after their fabrication at $25^{\circ} \mathrm{C}$ in the dry state. Five specimens were tested for each sample. Values are expressed as means \pm standard error.

In a second set of experiments samples were measured after storage in $\mathrm{SBF}$ medium at $37^{\circ} \mathrm{C}$ for a defined period of time. Before measuring the wet samples were dried to constant weight. Samples were run in duplicate.

\section{Cell cultivation studies}

Cell cultivation experiments were carried out in a growth chamber of a perfusion system operating at a continuous volume flow of $1.125 \mathrm{ml} / \mathrm{h}$. A Dulbecco's modified Eagle's medium (DMEM) containing $10 \%$ fetal calf serum (fcs), 100 units/ml penicillin, $100 \mathrm{mg} / \mathrm{ml}$ streptomycin, 10 $\mathrm{nM}$ dexamethasone and $10 \mathrm{nM}$ ascorbic acid-2-phosphate was used. Instead of conventional cultivation under 5\% $\mathrm{CO}_{2}$, the culture solution was supplemented by $2.5 \%$ HEPES buffer. Cultivation was performed over a period of 17 days in all experiments. Discs of the fabricated scaffolds with a diameter of $10 \mathrm{~mm}$ and a thickness of $4 \mathrm{~mm}$ were employed and sterilized by $\gamma$ irradiation ( $5 \mathrm{kGray}$ ) before use. Primary human osteoblasts (HCC-3001, Cell Lining, Berlin, Germany) and equine osteoblasts from the tuber coxae of a 10 years old horse were used. The cells seeded on the discs in a concentration of $3 \times 10^{5}$ cells per disc. Seeding was performed dynamically using a cell suspension. To initiate mineralization, the medium was supplemented with $1 \%$ of a $500 \mathrm{mM}$ phosphate solution for the last 3 days.

After harvesting, scaffolds were extensively rinsed with phosphate buffered saline (PBS) and fixed in 4\% neutral buffered formalin at $4^{0} \mathrm{C}$ for 24 hours. Sample materials were dehydrated, embedded in paraffin and sectioned ( 8 $\mathrm{mm}$ thick). After deparaffination, scaffold sections were stained with the von Kossa technique to show calcification. Toluidine blue stain and Masson-Goldner stain were used to assess proteoglycan accumulation and collagen accumulation, respectively. Scaffold analysis and content of glycosaminoglycans and collagens was controlled by light microscopy. Images were documented by a Polaroid (Waltham, MA, USA) MicroCam system.

Selected scaffolds with and without cells were processed for scanning electron microscopy (SEM). SEM was carried out after glutaraldehyde fixation, ethyl alcohol dehydration and gold sputtering using established techniques.

\section{Alkaline Phosphatase activity}

Alkaline Phosphatase (ALP) activity was measured by the method of Beresford et al. (1986) at days 6 and 12 of the cultivation using a commercially available kit (Sigma Diagnostics, St. Louis, MO, USA). Scaffolds without cells serves as control. ALP activity was expressed as units per $\mathrm{mg}$ protein. Total protein content was determined spectrophotometrically according to Bradford (1976) using the Bio Rad Protein Assay (Bio-Rad Laboratories, München, Germany).

\section{Results and Discussion}

\section{Synthesis of the scaffold components}

Oligo-L-lactides of different molecular weight possessing two terminal hydroxyl groups were prepared by ring-opening oligomerization of L-lactide in the presence of dianhydro-D-glucitol as initiator and stannous ethylhexanoate as a catalyst (Fig. 1). Dianhydro-D-glucitol represents a difunctional, biocompatible initiator derived from the natural monosaccharide D-glucose. It possesses a sufficient thermal stability in the temperature range of $140-150^{\circ} \mathrm{C}$, where the oligomerization reaction occurs and because of its low melting point $\left(63^{\circ} \mathrm{C}\right)$, the reaction can be performed in the melt under homogeneous conditions.

The molecular weights of the synthesized oligo-Llactides were controlled by the employed monomer : initiator ratios. Oligo-L-lactides with 2, 4 and 6 lactyl units per hydroxylic group of dianhydro-D-glucitol were prepared. The obtained oligomers are colourless, viscous liquids at room and body temperature.

GPC measurements of the oligomers (Table 1) show close agreement between the determined molecular weights

Table 1. Molecular weights of the synthesized oligo-Llactides determined by GPC.

\begin{tabular}{|c|c|c|c|}
\hline \multirow[t]{2}{*}{ Oligomer } & \multirow[t]{2}{*}{$n^{1)}$} & \multicolumn{2}{|c|}{ Molecular weight [gmol $\left.^{-1}\right]$} \\
\hline & & calculated $^{2)}$ & found \\
\hline DL-01 & 2 & 434 & 419 \\
\hline DL-02 & 4 & 722 & 674 \\
\hline DL-03 & 6 & 1011 & 981 \\
\hline
\end{tabular}

1) number of lactyl repeating units per $\mathrm{OH}$ group of the initiator

2) calculated on the basis of the used monomer : initiator ratio. 


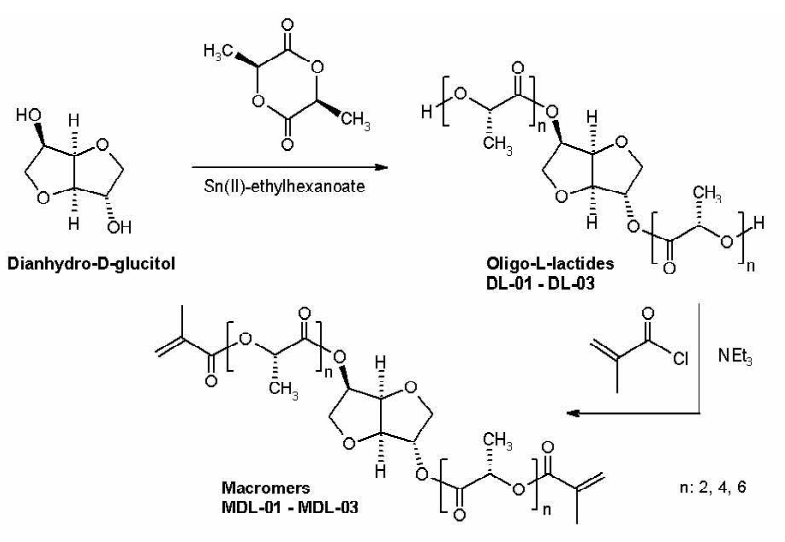

Figure 1. Synthesis of oligo-L-lactide macromers.

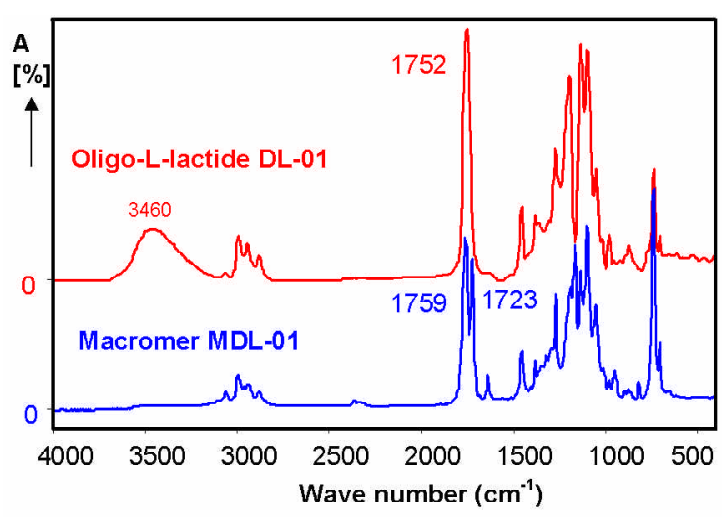

Figure 2. FT-IR spectra ( $\mathrm{KBr}$ technique) of the oligoL-lactide DL-01 and the corresponding macromer MDL01 . and the theoretical values calculated from monomer : initiator ratios. In the next step, macromers were synthesized by reaction of the oligo-L-lactides with methacryloyl chloride in dichloromethane using triethylamine as acid scavenger.

After careful purification of the raw products by extraction, the macromers were obtained as yellowish low to highly viscous liquids at room temperature. The pure macromers can be stored in a refrigerator for several months without spontaneous polymerization. They are soluble in various organic solvents (e. g. dichloromethane) and miscible with conventional methacrylate monomers like methylmethacrylate (MMA) or 2-hydroxyethyl methacrylate (HEMA).

Conventional spectroscopic techniques were used for the structural characterization of the prepared oligomers. The complete conversion of the terminal hydroxyl groups in the oligo-L-lactides was revealed by Fourier-Transform Infra-Red (FT-IR) spectroscopy (Fig. 2). In the IR spectra of the macromers, the characteristic absorption for the lactide ester groups between 1758 and $1765 \mathrm{~cm}^{-1}$ and an additional absorption at $1727 \mathrm{~cm}^{-1}$ due to the methacrylate groups can be observed. The absorption for the free hydroxyl groups of the oligolactides between 3300 and 3600 $\mathrm{cm}^{-1}$ has disappeared confirming complete substitution of the hydroxyl groups in the macromers by methacrylate functions.

\section{Fabrication and properties of the highly porous scaffolds}

Mixtures comprising one of the prepared oligolactide macromers and a conventional radical initiation system (e. g. dibenzoyl peroxide/ $p$-dimethyltoluidine) readily polymerize at room or body temperature to form solid polymer networks.

Based on these findings, curable mixtures for the scaffold fabrication were developed. The composition of the curable mixtures were systematically varied using various inorganic and organic filler materials $\left(\mathrm{CaCO}_{3}\right.$, anhydrous $\mathrm{CaHPO}_{4}$, poly-D,L-lactide). Dibenzoyl peroxide was employed as initiator and acetone was tested as diluent to modulate the viscosity of the mixtures. Optimized compositions of the curable mixtures are given in Table 2 .

A self-prepared porogen matrix of lump sugar was used to generate an open pore structure. The porogen matrices were soaked with an appropriate curable mixture and the filled devices were cured at $100^{\circ} \mathrm{C}$. It should be mentioned, that the cured, compact devices can be processed and shaped by conventional mechanical techniques like drilling, grinding or sawing.

After leaching out the porogen with boiling water,

Table 2. Composition of the curable mixtures for the scaffold fabrication ( $4.0 \mathrm{wt} .-\%$ of dibenzoyl peroxide related to the macromer weight was used as initiator).

\begin{tabular}{|l|l|c|c|c|}
\hline Curable mixture & Macromer (wt.-\%) & Comonomer (wt.-\%) & Filler (wt.-\%) & Diluent (wt.-\%) \\
\hline CM-01 & MDL-01 (82.50) & - & - & acetone (17.50) \\
CM-02 & MDL-03 (69.92) & - & - & acetone (30.08) \\
CM-03 & MDL-01 (82.5) & MMA (17.50) & - & - \\
CM-04 & MDL-01 (68.75) & MMA (14.58) & - & acetone (16.67) \\
CM-05 & MDL-01 (43.42) & - & $\mathrm{CaCO}_{3}(26.32)$ & acetone (30,26) \\
CM-06 & MDL-01 (56.90) & MMA (12.07) & $\mathrm{CaCO}_{3}(17.24)$ & acetone $(13.79)$ \\
CM-07 & MDL-01 (43.42) & - & $\mathrm{CaHPO}_{4}(26.32)$ & acetone $(30.26)$ \\
CM-08 & MDL-01 (56.90) & - & $\operatorname{PDLL~}(17.24)^{\text {acetone }(25.86)}$ \\
\hline
\end{tabular}

PDLL: poly-D,L-lactide (molecular weight: 17,000 gmol $^{-1}$ ) 
Table 3. Porosities of the fabricated scaffolds.

\begin{tabular}{|l|l|l|l|}
\hline Sample & \multicolumn{2}{|c|}{ Density [gcm $\left.{ }^{-3}\right]$} & Porosity \\
& Scaffold & Polymer & [\%] \\
\hline CM-01 & 0.206 & 1.335 & 85 \\
CM-02 & 0.196 & 1.180 & 83 \\
CM-03 & 0.265 & 1.347 & 80 \\
CM-04 & 0.220 & 1.310 & 83 \\
CM-05 & 0.231 & 1.225 & 81 \\
CM-06 & 0.209 & 1.413 & 85 \\
CM-07 & 0.215 & 1.312 & 84 \\
CM-08 & 0.190 & 1.266 & 86 \\
\hline
\end{tabular}

highly porous and dimensionally stable scaffolds were obtained.

Scaffold samples leached out for $16 \mathrm{~h}$ were milled and extracted to determine residual porogen in the matrices by conventional analytical methods. Only traces of sucrose could be found in the extracts. Thus, a leaching time of $16 \mathrm{~h}$ should be sufficient to ensure removal of the porogen. The porosities of the scaffolds derived from different chemical compositions were determined by measuring the apparent density of the scaffolds and the densities of the corresponding solid polymeric materials. Based on these density measurements the porosities of the scaffolds were calculated to range between 80 and $86 \%$ (Table 3 ).

Scanning electron microscopic investigations revealed a foam-like scaffold structure consisting in a polymer or composite skeleton with micropores throughout and evenly distributed interconnected macropores of irregular shape (Fig. 3).

The size of the macropores ranges from 150 up to 400 $\mu \mathrm{m}$ and correlates with the size distribution of the saccharose crystals in the porogen matrix. Saccharose crystals of the porogen matrix are not distinguishable any more in the scaffolds. The variation of the composition of the curable mixtures as given in Table 1 has no detectable effect on the morphology and the porosity of the formed scaffolds.

The in vitro biodegradation behaviour of the fabricated scaffolds was studied in SBF medium at $37^{\circ} \mathrm{C}$ by measuring the weight loss of the scaffolds as a function of the storage time. A continuous course of degradation has been observed for the prepared scaffolds as it can be seen in Figure 4 for the sample CM-02. The rate of degradation can be influenced by the used additives (comonomer, filler, diluent) as shown in Figures 5 and 6.

The use of MMA, a common monomer in bone cements, as comonomer also gave degradable scaffolds (Fig. 5, sample CM-04) but as expected, the rate of degradation is significantly decreased compared to those of the scaffolds produced only from oligo-L-lactide macromers (sample CM-01).

In Fig. 6 the degradation behaviour of the composite scaffolds containing calcium carbonate and calcium hydrogen phosphate is depicted. The addition of calcium carbonate to the oligolactide macromer mixture (sample CM-
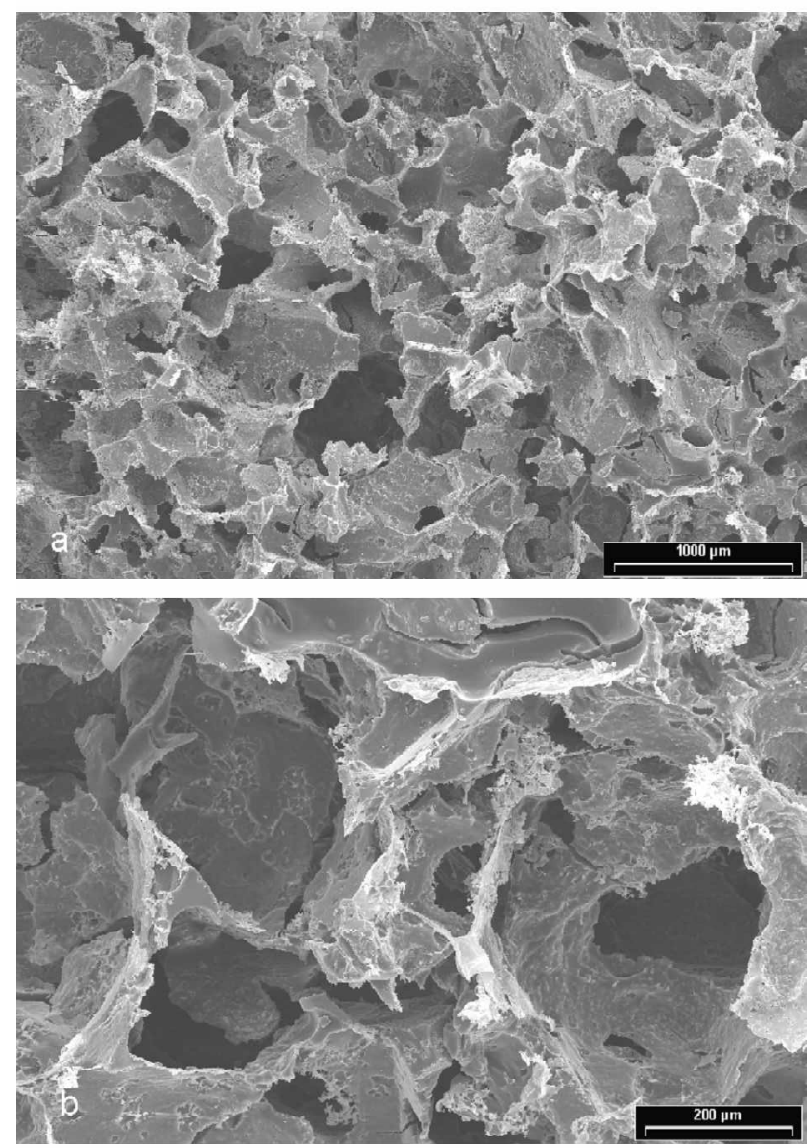

Figure 3. Scanning electron micrograph of scaffolds fabricated from CM-06: (a) bar $=1000 \mu \mathrm{m}$, (b) bar $=$ $200 \mu \mathrm{m}$.

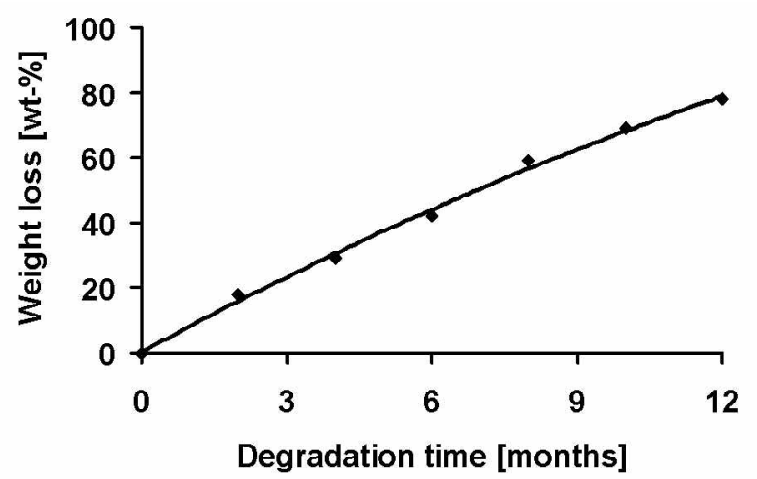

Figure 4. Weight loss of the scaffold CM-02 during the storage in SBF medium at $37^{\circ} \mathrm{C}$.

05) has relatively little effect on the rate of degradation (for comparison see sample CM-01 without filler in Fig. 5). With calcium hydrogen phosphate as filler material the degradation occurs somewhat slower compared to calcium carbonate.

The scaffold fabrication procedure results in highly porous but dimensionally stable devices.

As a parameter for the mechanical stability of the scaffolds, their compression strength was measured in the dry state directly after preparation (Table 4).

The highest values for the compression strength were obtained with the scaffolds prepared without organic or 


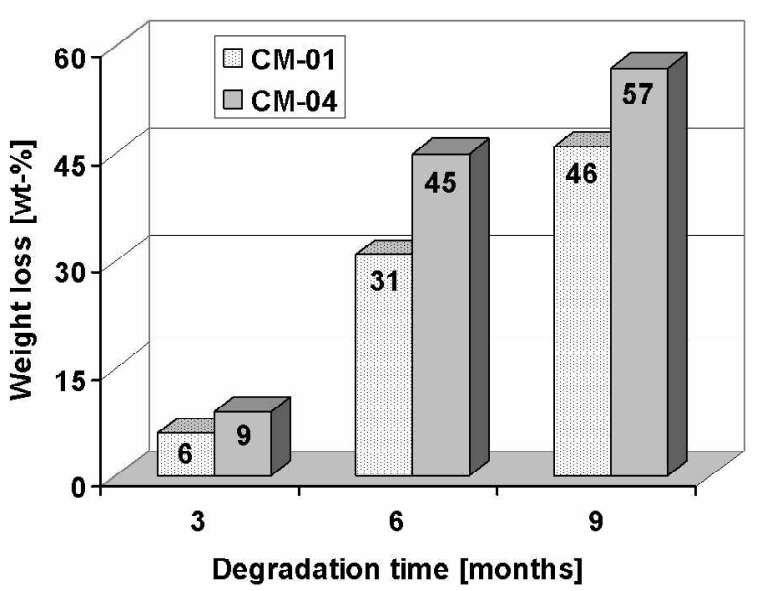

Figure 5. Comparison of the degradation behaviour of the scaffolds CM-01 and CM-04 in SBF medium at $37^{\circ} \mathrm{C}$.

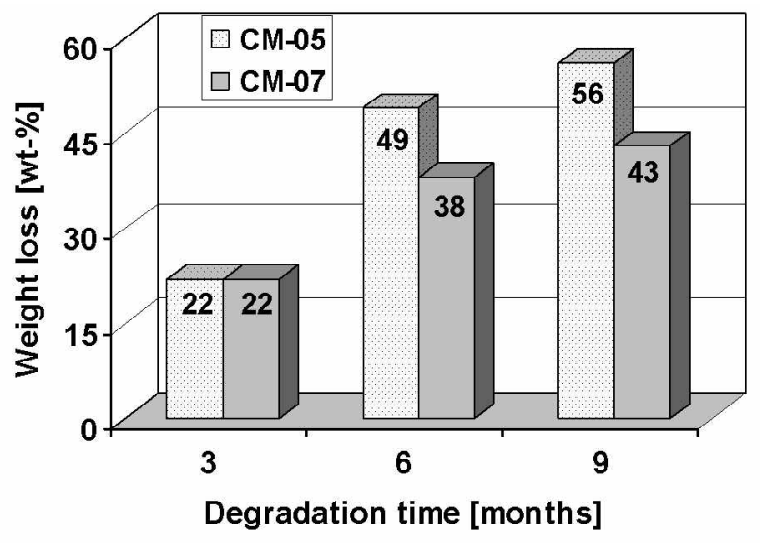

Figure 6. Comparison of the degradation behaviour of the scaffolds CM-05 and CM-07 in SBF medium at $37^{\circ} \mathrm{C}$.

inorganic fillers but with MMA as comonomer.

The compression strength of representative scaffolds during the degradation course in SBF medium is illustrated in Figure 7. A continuous relatively slow drop of the compression strength of the scaffolds can be observed over the studied degradation period of 7 months. As expected the addition of MMA as comonomer to the macromer mixture (sample CM-04) leads to scaffolds with higher mechanical strength.

Degradation studies were done by looking at the mechanical properties and the weight loss of the scaffolds as a function of the storage time in SBF medium. The determination of the reduction of molecular weights of the polymeric components by GPC analysis is a further valuable method to study the course of degradation. Unfortunately, after the formation of the polymeric networks and the scaffold fabrication, GPC analysis is not possible because the scaffold materials (polymer networks) are not soluble in organic solvents usable in GPC. There would be the possibility to study the occurence of soluble products (e.g. lactic acid or its oligomers) during the degradation process by chromatographic techniques like GPC or high performance liquid chromatography (HPLC). The development of a suitable method is now in progress. On the other hand,
Table 4. Compression strength of the scaffolds measured in the dry state at $25^{\circ} \mathrm{C}$.

\begin{tabular}{|l|c|}
\hline Sample & Compression strength [MPa] \\
\hline CM-01 & $0.30 \pm 0.09$ \\
CM-02 & $0.35 \pm 0.17$ \\
CM-03 & $1.08 \pm 0.12$ \\
CM-04 & $0.73 \pm 0.11$ \\
CM-05 & $0.51 \pm 0.08$ \\
CM-06 & $0.27 \pm 0.05$ \\
CM-07 & $0.36 \pm 0.04$ \\
CM-08 & $0.18 \pm 0.08$ \\
\hline
\end{tabular}

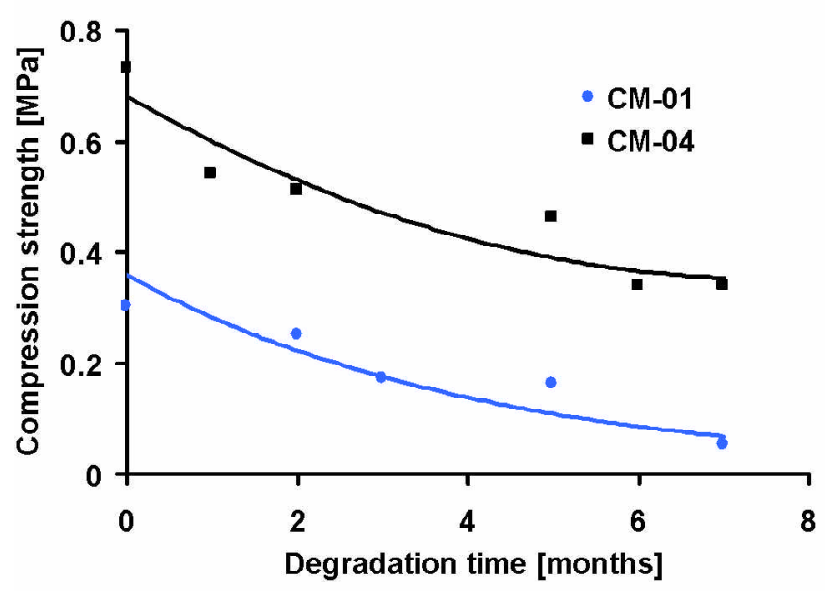

Figure 7. Effect of degradation on the compression strength of the scaffold materials CM-01 and CM-04, resp., in SBF medium at $37^{\circ} \mathrm{C}$.

based on our degradation studies over a period of 12 months, a peak release of lactic acid in large amounts should not be expected.

\section{In vitro cell cultivation}

Primary human osteoblasts were seeded on fabricated and sterilized discs of the scaffold materials. Although polylactide surfaces are known to be rather hydrophobic, a relatively high cell adhesion on the tested materials was observed even without additional hydrophilic coating of the scaffold surface.

The seeded scaffolds have been incubated for 17 days in a continuously working perfusion system using a conventional DMEM cell culture medium. It could be demonstrated by SEM investigations that after this time the scaffolds were completely overgrown by osteoblasts. Most of the outer macropores became sealed off by a continuous layer of cells (Fig. 8a). While some of the cells adhered on the macropore surface, others spanned the pores in a threedimensional fashion, surrounded by a fibrous extracellular matrix (Fig. 8b). Similar results (not shown) were obtained seeding equine osteoblasts from the tuber coxae on the prepared scaffolds. Toluidine blue stained histological cross-sections from slides of the incubated discs showed compact osteoide like areas inside the discs and especially 

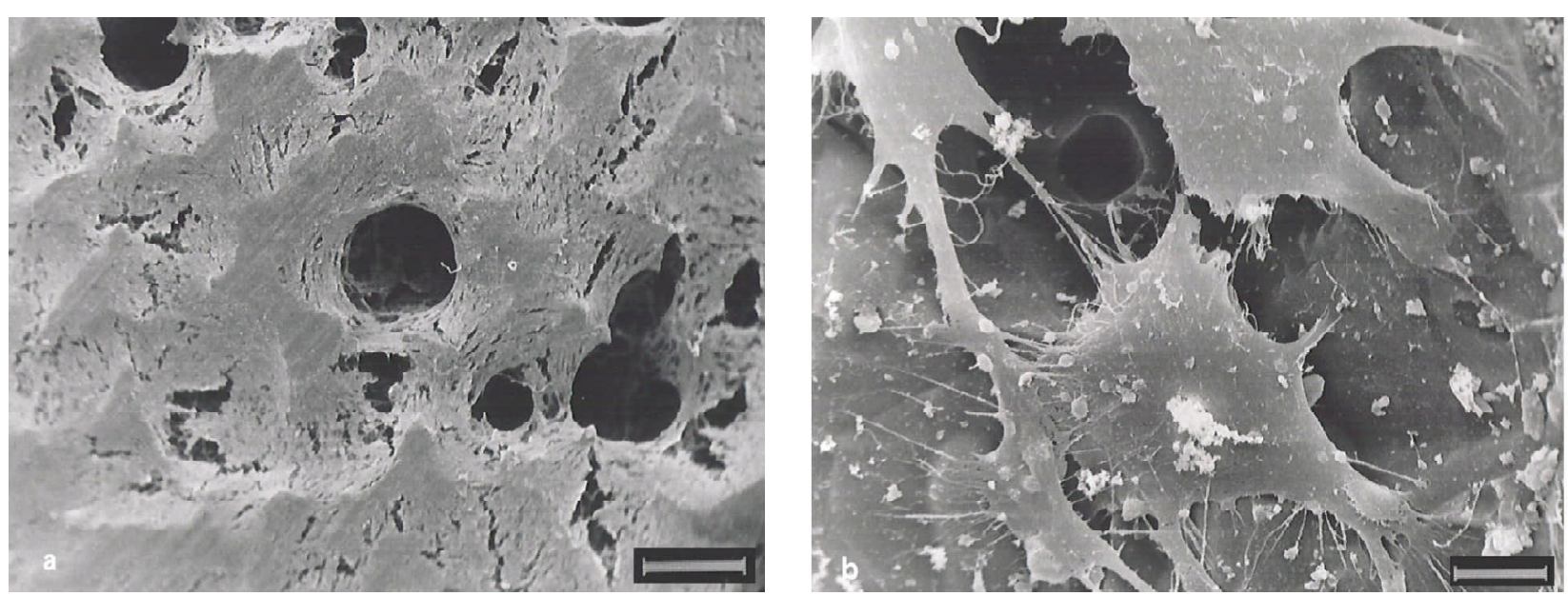

Figure 8. Scanning electron micrograph of scaffolds (CM-06) after cultivation with osteoblasts over a period of 17 days: (a) bar $=500 \mu \mathrm{m}$, (b) bar $=10 \mu \mathrm{m}$
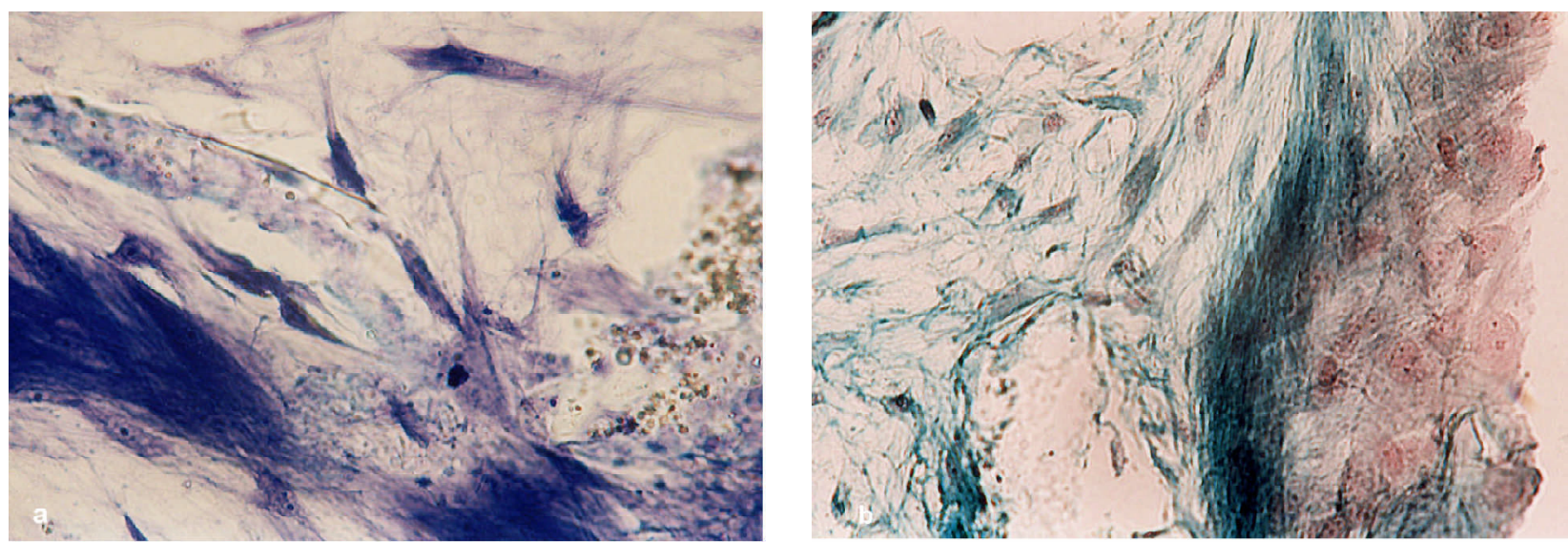

Figure 9. Histological cross-sections of scaffolds (CM-06) seeded with human osteoblasts: (a) toluidine blue stained cross-section (magnification 400 fold), (b) Masson-Goldner stained cross section (magnification 400 fold).

along their borders (Fig. 9a). It could be demonstrated by von Kossa staining that such areas had already been started to mineralize. Toluidine blue staining also exhibits clear and distinct proteoglycan biosynthesis, especially in densely packed cell layers of the surface area of the scaffolds. Sometimes cells were surrounded by lacunae. Also MassonGoldner stains (Fig. 9b) showed clear collagen biosynthesis. As in the toluidine blue stains collagen was densely packed in cell layers of the surface area.

Differentiated function of the osteoblasts was assessed by monitoring their ALP activity. The ALP assay was carried out during the cultivation study after 6 and 12 days. The determined values for the ALP are shown in Figure 10. Compared to the control, a distinct ALP activity of the osteoblasts seeded on the prepared scaffolds can be detected.

\section{Conclusions}

Bone regeneration applying novel techniques of tissue engineering requires biocompatible and preferably resorbable materials providing a framework for cells to adhere, proliferate and create extracellular matrix.

Oligolactide derived macromers which are able to form polymeric networks represent valuable starting materials for the fabrication of highly porous scaffolds usable in bone tissue engineering. Based on these macromers, a simple and reproducible scaffold fabrication process consisting in a curing and a leaching step was developed. Using this process, dimensionally stable scaffolds with an interconnective, open pore structure and pore sizes up to a few hundred microns were created. Porosities between 80 and $86 \%$ were achieved. Various biodegradable as well as osteoconductive components like polylactide, calcium carbonate or calcium phosphates including hydroxyapatite, can be added during the fabrication process to modulate the properties of the resulting scaffolds. The scaffolds available in various shapes and sizes can be processed by conventional mechanical techniques. Compared to pure polylactide scaffolds of similar pore structure, the ones prepared from oligolactide macromers exhibit a significantly higher mechanical stability. A further increase in the mechanical strength can be achieved by the use of conventional biocompatible methacrylates like MMA or HEMA as comonomers.

The in vitro degradation behaviour of scaffolds with different compositions was studied in SBF medium revealing a continuous weight loss of all of the tested scaffold materials over a degradation period of up to 12 months. As expected, the rate of degradation depends on the cross- 


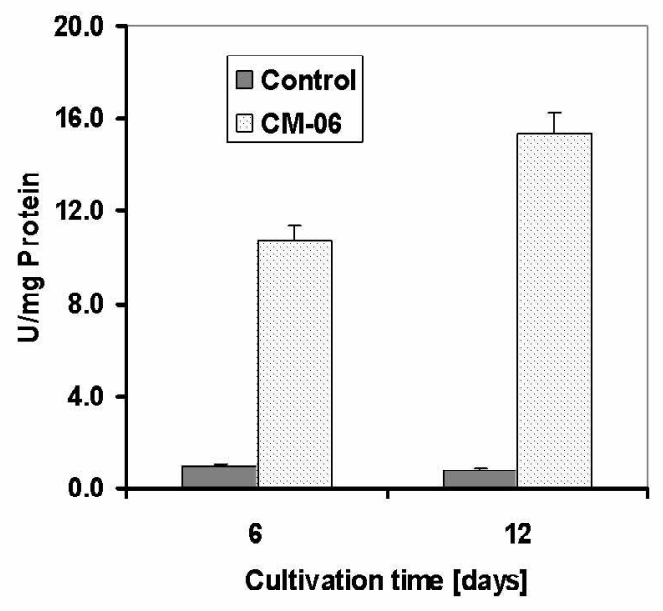

Figure 10. ALP activity of osteoblasts seeded onto CM-06.

linking density, the nature of added comonomers and further additives as well.

First in vitro cell cultivation experiments demonstrated an excellent biocompatibility of the fabricated scaffolds and their potential to serve as biodegradable matrices for bone tissue engineering. Future studies involve the optimization of tissue growth within the porous matrices and in vivo testing of the scaffold materials.

\section{Acknowledgements}

The authors would like to thank R. Rasch, P. Schwartz (University of Göttingen), M. Frigge (INNOVENT Jena), and I. Ponomarev (Research Centre of Medical Technology and Biotechnology, Bad Langensalza) for scientific and technical assistance. This work was supported by the German Ministry of Economics.

\section{References}

Agrawal CM, Athanasiou KA, Heckman JD (1997) Biodegradable PLA-PGA polymers for tissue engineering in orthopaedics. Mater Sci Forum 250: 115-128.

Beresford JN, Gallagher JA, Russell RGG (1986) 1,25 Dihydroxyvitamin D3 and human bone derived cells in Vitro: Effects on alkaline phosphatase, type I collagen and proliferation. Endocrinology 119: 1776-1794.

Boyan BD, Lohmann CH, Romero J, Schwartz Z (1999) Bone and cartilage tissue engineering. Clin Plast Surg 26: 629-645.

Bradford MM (1976) A rapid and sensitive method for the quantition microgram quantities of protein utilizing the principle of protein-dye binding. Anal Biochem 72: 248254.

Burg KJL, Porter S, Kellam JF (2000) Biomaterial developments in bone tissue engineering. Biomaterials 21: 2347-2359.

Cho SB, Nakanishi K, Kokubo T, Soga N, Ohtsuki C, Nakamura T, Kitsugi T, Yamamuro T (1995) Dependence of apatite formation on silica gel on its structure: effect of heat treatment. J Am Ceram Soc 78: 1769-1774.
Fleming JE, Cornell CN, Muschler GF (2000) Bone cells and matrices in orthopedic tissue engineering. Orthop. Clinics North America 31: 357-374.

Griffith LG, Naughton G (2002) Tissue EngineeringCurrent Challenges and Expanding Opportunities. Science 295: 1009-1014.

Han DK, Hubbell JA (1997) Synthesis of polymer network scaffolds from L-lactide and poly(ethylene glycol) and their interaction with cells. Macromolecules 30: $6077-$ 6083.

Hench LL, Polak JM (2002) Third-Generation Biomedical Materials. Science 295: 1014-1017.

Hutmacher DW (2000) Scaffolds in tissue engineering bone and cartilage. Biomaterials 21: 2529-2543.

Jackson DW, Simon TM (1999) Tissue engineering principles in orthopaedic surgery. Clin Orthop Relat Res 367S: S31-S45.

Lee YM, Kim SS (1997) Hydrogels of poly(ethylene glycol)-co-poly(lactones) diacrylate macromers and $\beta$ chitin. Polymer 38: 2415-2420.

Lendlein A, Neuenschwander P, Suter UW (1998) Tissue-compatible multiblock copolymers for medical applications, controllable in degradation rate and mechanical properties. Macromol Chem Phys 199: 2785-2796.

Luyten FP, Dell'Accio F, De Bari C (2001) Skeletal tissue engineering: opportunities and challenges. Best Pract \& Res. Clin Rheumatol 15: 759-770.

Middleton JC, Tipton AJ (2000) Synthetic biodegradable polymers as orthopedic devices. Biomaterials 21: 2335-2346.

Perrin DD, Amarego WLF (1988) Purification of laboratory chemicals. Pergamon Press, Oxford. pp. 1-56.

Simske SJ, Ayers RA, Bateman TA (1997) Porous materials for bone engineering. Mater Sci Forum 250: 151182.

Storey RF, Warren SC, Allison CJ, Wiggins JS, Puckett AD (1993) Synthesis of bioabsorbable networks from methacrylate-endcapped polyesters. Polymer 34: 43654372 .

Thomson RC, Yaszemski MJ, Powers JM, Mikos AG (1995) Fabrication of biodegradable polymer scaffolds to engineer trabecular bone. J Biomater Sci Polymer Edn 7: 23-38.

Wenz R, Nies B (1996) Bioresorbierbare Polymerisationsprodukte aus strahlungshärtbaren Bindemittelsystemen (Bioresorbable polymerization products from radiation-curable binder systems). German Patent No. 19646782.

\section{Discussion with Reviewers}

H. Winet: What was your reason for supplementing the culture solution with HEPES?

Authors: The reason for supplementing the culture solution with HEPES was to avoid $\mathrm{pH}$ shifts in the micro environment between the cells and the scaffold.

H. Winet: It is not clear from your description if any cells survived at the core of the scaffold. Figure 8a shows some 
pores unsealed. How deep did the cells grow into them? Authors: We found that during the entire cultivation period the cells survived at the core of the scaffolds. As shown in Figure 8a some pores had been unsealed by cells. Possibly this is an effect of the pore size. Cells grew into the porous scaffolds up to a depth of about $2 \mathrm{~mm}$.

H. Winet: No numbers are given for cell density. Was Figure $8 \mathrm{~b}$ representative?

Authors: Figure $8 \mathrm{~b}$ is representative. With the exception of very few regions around unsealed pores, the scaffolds were covered by cells to an extent of 90-100 \%.

H. Winet: How thick was the fibrous capsule?

Authors: The thickness of the fibrous capsule varied between 100 and $200 \mu \mathrm{m}$.

H. Winet: How do your results compare with those from poly $(\alpha$-hydroxy acid) scaffolds?

Authors: We prepared highly porous scaffolds from pure poly(L-lactide) by the same method as applied for the described scaffolds. Compared to our polymer network scaffolds, these porous non-crosslinked poly(L-lactide) scaffolds are very fragile and difficult to handle. Therefore we have not used these polylactide scaffolds in cell culture experiments. From literature data and our own experiences with commercial polylactide fleeces, we would assume that both polymeric materials show similar properties as matrices in osteoblast cultivation concerning cell adhesion, growth and differentiated function.

M. A. Barbosa: The paper shows that the highest compression strengths were obtained with scaffolds prepared without organic or inorganic fillers. How does the compression strength of the composites compare with that of the filler materials? How does the interface between the polymer and the filler behave during compression, i.e. is there detachment or fracture of one of the phases?

Authors: In fact, the highest compression strengths were obtained with scaffolds prepared with MMA as comonomer and without fillers $\left(\mathrm{CaCO}_{3}, \mathrm{CaHPO}_{4}, \mathrm{PDLL}\right)$. It was not possible to produce comparable scaffolds from one of the pure inorganic filler materials to measure the corresponding compression strength. The compression strength of pure PDLL scaffolds prepared by the described procedure was much lower than those found for the polymer network scaffolds. Because of the highly porous structure of the scaffolds, a quantitative analysis of the breaking behaviour is quite difficult, but in most cases we observed both phase detachment and fracture of the filler grains during the compression of the scaffolds. 